International Mathematical Forum, Vol. 9, 2014, no. 1, 1 - 11

HIKARI Ltd, www.m-hikari.com

http://dx.doi.org/10.12988/imf.2014.311222

\title{
All-Derivable Subsets for Nest Algebras on Banach Spaces
}

\author{
Yanfang Zhang, Jinchuan Hou ${ }^{1}$ \\ Department of Mathematics \\ Taiyuan University of Technology \\ Taiyuan 030024, P.R. China \\ sxzyf1012@sina.com, jinchuanhou@aliyun.com \\ Xiaofei Qi ${ }^{2}$ \\ Department of Mathematics \\ Shanxi University \\ Taiyuan 030006, P.R. China \\ xiaofeiqisxu@aliyun.com
}

Copyright (c) 2014 Yanfang Zhang, Jinchuan Hou and Xiaofei Qi. This is an open access article distributed under the Creative Commons Attribution License, which permits unrestricted use, distribution, and reproduction in any medium, provided the original work is properly cited.

\begin{abstract}
Let $\mathcal{N}$ be a nest on a complex Banach space $X$ and let $\operatorname{Alg} \mathcal{N}$ be the associated nest algebra. We say that a subset $\mathcal{S} \subset \operatorname{Alg} \mathcal{N}$ is an all-derivable subset of $\operatorname{Alg} \mathcal{N}$ if every linear map $\delta$ from $\operatorname{Alg} \mathcal{N}$ into itself derivable on $\mathcal{S}$ (i.e. $\delta$ satisfies that, for each $Z \in \mathcal{S}, \delta(A) B+A \delta(B)=$ $\delta(Z)$ for any $A, B \in \operatorname{Alg} \mathcal{N}$ with $A B=Z$ ) is a derivation. In this paper, we show that $\mathcal{S}$ is an all-derivable subset of $\operatorname{Alg} \mathcal{N}$ if $\operatorname{span}\{\operatorname{ran}(Z): Z \in$ $\mathcal{S}\}$ is dense in $X$ or $\cap\{\operatorname{ker} Z: Z \in \mathcal{S}\}=\{0\}$.
\end{abstract}

Mathematics Subject Classification: 47B47, 47L35

Keywords: Banach spaces, nest algebras, derivations, all-derivable subsets

\footnotetext{
${ }^{1}$ Supported by NNSFC $11171249,11271217$.

${ }^{2}$ Supported by NNSFC 11101250 and YSFSPC 2012021004.
} 


\section{Introduction}

A linear map $\delta: \mathcal{A} \rightarrow \mathcal{A}$, where $\mathcal{A}$ is an algebra, is a derivation if $\delta(A B)=$ $\delta(A) B+A \delta(B)$ for all $A, B \in \mathcal{A}$. The derivations are very important linear maps both in theory and applications, and were studied intensively. Particularly, the question of under what conditions that a linear (even additive) map becomes a derivation attracted much attention of authors (for instance, see $[2,5,6,10]$ and the references therein). One approach is to characterize derivations by their local behaviors. We say that a map $\varphi: \mathcal{A} \rightarrow \mathcal{A}$ is derivable at a point $Z \in \mathcal{A}$ if $\varphi(A) B+A \varphi(B)=\varphi(Z)$ for any $A, B \in \mathcal{A}$ with $A B=Z$, and we call such $Z$ a derivable point of $\varphi$. Obviously, a linear map is a derivation if and only if it is derivable at every point. It is natural and interesting to ask the question whether or not a linear map is a derivation if it is derivable only at one given point. As usual, we say that an element $Z \in \mathcal{A}$ is an all-derivable point of $\mathcal{A}$ if every linear map on $\mathcal{A}$ derivable at $Z$ is in fact a derivation. So far, we have known that there exist many all-derivable points (or full-drivable points) for certain (operator) algebras (see $[1,4,7,8,13,14,16]$ and the references therein). However, zero point 0 is not an all-derivable point for any algebra because the generalized derivations are derivable at 0 [8].

The invertible elements are all-derivable points for many algebras. For instance, invertible elements are all-derivable points of $\mathcal{J}$-subspace lattice algebras $([4,9])$. As nest algebras are of an important class of operator algebras, there are many papers on finding all-derivable points of nest algebras. But so far all known results were obtained under some additional assumptions on nests or the spaces (Ref. $[1,8,15])$. The problem of finding all-derivable points of any nest algebras on any Banach spaces was attacked by [12] recently. It was shown in [12] that every injective operator as well as every operator of dense range is an all-derivable point of any nest algebra on any Banach space.

More generally, we may introduce a notion of derivable subsets. Let $\mathcal{A}$ be an algebra and $\delta: \mathcal{A} \rightarrow \rightarrow \mathcal{A}$ be a linear map. If a subset $\mathcal{S} \subset \mathcal{A}$ satisfies that every $Z \in \mathcal{S}$ is a derivable point of $\delta$, we say that $\mathcal{S}$ is a derivable subset of $\delta$ or $\delta$ is derivable on $\mathcal{S}$; if, for any linear map $\varphi: \mathcal{A} \rightarrow \mathcal{A}, \varphi$ is derivable on $\mathcal{S}$ will imply that $\varphi$ is a derivation, we say that $\mathcal{S}$ is an all-derivable subset of the algebra $\mathcal{A}$. Clearly, a derivation is derivable on any subset.

The purpose of the present paper is, based on the study in [12], to find some all-derivable subsets for all nest algebras on complex Banach spaces without any additional assumptions on the nests, and generalize the main result obtained in [12].

The following is the main result of this paper.

Theorem 1.1. Let $\mathcal{N}$ be a nest on a complex Banach space $X$ with $\operatorname{dim} X \geq 2$ and $\mathcal{S} \subset \operatorname{Alg} \mathcal{N}$. If $\cap\{\operatorname{ker} Z: Z \in \mathcal{S}\}=\{0\}$ or if $\operatorname{span}\{\operatorname{ran}(Z)$ : $Z \in \mathcal{S}\}$ is dense in $X$, then $\mathcal{S}$ is an all-derivable subset of the nest algebra 
$\operatorname{Alg} \mathcal{N}$, that is, a linear map $\delta: A \lg \mathcal{N} \rightarrow \operatorname{Alg} \mathcal{N}$ is derivable on the subset $\mathcal{S}$ if and only if $\delta$ is a derivation.

Particularly, as a consequence we obtain the main result in [12] again by taking $\mathcal{S}=\{Z\}$, the singleton consisting of $Z$, where $Z$ is injective or of dense range.

Corollary 1.2. Let $\mathcal{N}$ be a nest on a complex Banach space $X$ with $\operatorname{dim} X \geq 2$ and $\delta: \operatorname{Alg} \mathcal{N} \rightarrow \operatorname{Alg} \mathcal{N}$ be a linear map. Let $Z \in \operatorname{Alg} \mathcal{N}$ be an injective operator or an operator with dense range. Then $\delta$ is derivable at the operator $Z$ if and only if $\delta$ is a derivation.

The following corollary is also immediate.

Corollary 1.3. Let $\mathcal{N}$ be a nest on a complex Banach space $X$ with $\operatorname{dim} X \geq 2$ and $\delta: \operatorname{Alg} \mathcal{N} \rightarrow \operatorname{Alg} \mathcal{N}$ be a linear map. Let $P \in \operatorname{Alg} \mathcal{N}$ be an idempotent operator. Then $\delta$ is derivable at both $P$ and $I-P$ if and only if $\delta$ is a derivation.

The paper is organized as follows. We fix some notations and preliminary lemmas in Section 2 and prove Theorem 1.1 in Section 3.

\section{Preliminaries and lemmas}

In this section let us fix some notations and give some lemmas.

For a (real or complex) Banach space $X$, denote by $X^{*}$ and $\mathcal{B}(X)$ the dual of and the algebra of all bounded linear operators on $X$, respectively. The canonical embedding from $X$ into $X^{* *}$ is denoted by $\kappa$. For any $T \in \mathcal{B}(X)$, $T^{*}$ stands for its adjoint operator which is the linear operator from $X^{*}$ into $X^{*}$ defined by $\left(T^{*} f\right)(x)=f(T x)$ for any $f \in X^{*}$ and $x \in X$. If $f \in X^{*}$ and $x \in X, x \otimes f$ stands for the operator defined by $(x \otimes f)(y)=f(y) x$ for any $y \in X$. It is easily seen that $\operatorname{rank}(x \otimes f) \leq 1 ; x \otimes f$ is of rank one if and only if both $x$ and $f$ are nonzero, and every rank one operator can be represented as this form. Clearly, $(x \otimes f)^{*}=f \otimes x^{* *}$ and $x^{* *} T^{*}=(T x)^{* *}$, where $x^{* *}=\kappa(x)$. For any non-empty subset $N \subseteq X$, denote by $N^{\perp}$ the annihilator of $N$, that is, $N^{\perp}=\left\{f \in X^{*}: f(x)=0\right.$ for every $\left.x \in X\right\}$. Some times we use $\langle x, f\rangle$ to present the value $f(x)$ of $f$ at $x$. In addition, we use the symbols $\operatorname{ran}(T)$ and $\operatorname{ker}(T)$ for the range and the kernel of operator $T$, respectively.

A nest $\mathcal{N}$ in $X$ is a complete chain of closed linear subspaces of $X$ containing the trivial subspaces $\{0\}$ and $X$, that is, any two elements in $\mathcal{N}$ can compare with each other under the partial order " $\subseteq$ " and $\mathcal{N}$ is closed under the formation of arbitrary closed linear span (denoted by $\bigvee$ ) and intersection (denoted by $\bigwedge$ ). Denote $\operatorname{Alg} \mathcal{N}=\{A \in \mathcal{B}(X): \mathcal{N} \subseteq \operatorname{Lat} A\}$ the associated nest algebra, where Lat $A$ stands for the invariant subspace lattice of $A$. When $\mathcal{N} \neq\{\{0\}, X\}$, we say that $\mathcal{N}$ is nontrivial. It is clear that $\operatorname{Alg} \mathcal{N}=\mathcal{B}(X)$ if $\mathcal{N}$ is trivial. For $N \in \mathcal{N}$, let $N_{-}=\bigvee\{M \in \mathcal{N} \mid M \subset N\}$, $N_{+}=\bigwedge\{M \in \mathcal{N} \mid N \subset M\}$ and $N_{-}^{\perp}=\left(N_{-}\right)^{\perp}$. Also, let $\{0\}_{-}=\{0\}$ and 
$X_{+}=X$. Denote $\mathcal{D}_{1}(\mathcal{N})=\bigcup\left\{N \in \mathcal{N} \mid N_{-} \neq X\right\}$ and $\mathcal{D}_{2}(\mathcal{N})=\bigcup\left\{N_{-}^{\perp} \mid N \in\right.$ $\mathcal{N}$ and $N \neq\{0\}\}$. Note that $\mathcal{D}_{1}(\mathcal{N})$ is dense in $X$ and $\mathcal{D}_{2}(\mathcal{N})$ is dense in $X^{*}$. It is clear that $\mathcal{D}_{1}(\mathcal{N})=X$ if and only if $X_{-} \neq X$, and $\mathcal{D}_{2}(\mathcal{N})=X^{*}$ if and only if $\{0\} \neq\{0\}_{+}$. It is well-known that a rank one operator $x \otimes f$ belongs to $\operatorname{Alg} \mathcal{N}$ if and only if there is some $N \in \mathcal{N}$ such that $x \in N$ and $f \in N_{-}^{\perp}$. For more information on nest algebras, we refer to $[3,11]$.

The following lemmas are needed to prove the main result.

Lemma 2.1-2.3 are easily checked.

Lemma 2.1. Let $\mathcal{A}$ be a unital ring. Suppose that $\delta: \mathcal{A} \rightarrow \mathcal{A}$ is an additive map. If nonzero element $Z \in \mathcal{A}$ is a derivable point of $\delta$, then, $\delta(I) Z=Z \delta(I)=0$.

Lemma 2.2. Let $\mathcal{A}$ be a unital ring. Suppose that $\delta: \mathcal{A} \rightarrow \mathcal{A}$ is an additive map. If nonzero element $Z \in \mathcal{A}$ is a derivable point of $\delta$, then,

(1) for every element $N \in \mathcal{A}$ with $N^{2}=0$, we have

$$
\delta(N Z)=\delta(N) Z+N \delta(Z)-\delta(I) N Z
$$

and

$$
[\delta(N) N+N \delta(N)-\delta(I)-N \delta(I) N] Z=0
$$

(2) for every element $N \in \mathcal{A}$ with $N^{2}=0$, we have

$$
\delta(Z N)=\delta(Z) N+Z \delta(N)-Z N \delta(I)
$$

and

$$
Z[\delta(N) N+N \delta(N)-\delta(I)-N \delta(I) N]=0
$$

Lemma 2.3. Let $\mathcal{A}$ be a unital ring. Suppose that $\delta: \mathcal{A} \rightarrow \mathcal{A}$ is an additive map. If nonzero element $Z \in \mathcal{A}$ is a derivable point of $\delta$, then, for any invertible element $A \in \mathcal{A}$,

$$
\delta\left(A^{-1} Z\right)=A^{-1} \delta(Z)-A^{-1} \delta(A) A^{-1} Z
$$

and

$$
\delta\left(Z A^{-1}\right)=\delta(Z) A^{-1}-Z A^{-1} \delta(A) A^{-1}
$$

Lemma 2.4. Let $\mathcal{A}$ be a unital complex algebra. Suppose that $\delta: \mathcal{A} \rightarrow \mathcal{A}$ is a linear map. If $Z \in \mathcal{A}$ is a nonzero element such that $\delta$ is derivable at $Z$, then the following are true.

(1) For any idempotent $P \in \operatorname{Alg} \mathcal{N}$, we have

$$
\delta(P Z)=\delta(P) Z+P \delta(Z)-\delta(I) P Z
$$


and

$$
\delta(P) Z=(\delta(P) P+P \delta(P)) Z
$$

(2) For any idempotent $P \in \operatorname{Alg} \mathcal{N}$, we have

$$
\delta(Z P)=\delta(Z) P+Z \delta(P)-Z P \delta(I)
$$

and

$$
Z \delta(P)=Z(\delta(P) P+P \delta(P))
$$

Proof. Let $P \in \mathcal{A}$ be any idempotent element, that is, $P$ satisfies $P^{2}-P$. Since $Z=\left(I-\frac{1-\sqrt{3} i}{2} P\right)\left(I-\frac{1+\sqrt{3} i}{2} P\right) Z$, we have

$$
\begin{aligned}
\delta(Z)= & \delta\left(I-\frac{1-\sqrt{3} i}{2} P\right)\left(I-\frac{1+\sqrt{3} i}{2} P\right) Z \\
& +\left(I-\frac{1-\sqrt{3} i}{2} P\right) \delta\left(Z-\frac{1+\sqrt{3} i}{2} P Z\right) \\
= & \delta(I) Z-\frac{1+\sqrt{3} i}{2} \delta(I) P Z-\frac{1-\sqrt{3} i}{2} \delta(P) Z+\delta(P) P Z+\delta(Z) \\
& -\frac{1+\sqrt{3} i}{2} \delta(P Z)-\frac{1-\sqrt{3} i}{2} P \delta(Z)+P \delta(P Z) .
\end{aligned}
$$

On the other hand, $Z=\left(I-\frac{1+\sqrt{3} i}{2} P\right)\left(I-\frac{1-\sqrt{3} i}{2} P\right) Z$ gives

$$
\begin{aligned}
\delta(Z)= & \delta(I) Z-\frac{1-\sqrt{3} i}{2} \delta(I) P Z-\frac{1+\sqrt{3} i}{2} \delta(P) Z+\delta(P) P Z+\delta(Z) \\
& -\frac{1-\sqrt{3} i}{2} \delta(P Z)-\frac{1+\sqrt{3} i}{2} P \delta(Z)+P \delta(P Z) .
\end{aligned}
$$

It follows from Eq.(2.1)-Eq.(2.2) that

$$
\delta(P Z)=\delta(P) Z+P \delta(Z)-\delta(I) P Z .
$$

Substituting Eq.(2.3) into Eq.(2.1) and notice that $\delta(I) Z=0$, one obtains

$$
\delta(P) Z=(\delta(P) P+P \delta(P)) Z,
$$

which completes the proof of (1).

By considering the equation

$$
Z=Z\left(I-\frac{1-\sqrt{3} i}{2} P\right)\left(I-\frac{1+\sqrt{3} i}{2} P\right)=Z\left(I-\frac{1+\sqrt{3} i}{2} P\right)\left(I-\frac{1-\sqrt{3} i}{2} P\right),
$$

one can get (2).

The following Lemmas 2.5-2.7 come from [12].

Lemma 2.5. Let $\mathcal{N}$ be a nest on a real or complex Banach space $X$ with $\operatorname{dim} X \geq 2$. Assume that $\delta: A \lg \mathcal{N} \rightarrow \operatorname{Alg} \mathcal{N}$ is a linear map satisfying $\delta(P)=\delta(P) P+P \delta(P)$ for all idempotent operators $P \in \operatorname{Alg} \mathcal{N}$ and $\delta(N) N+$ $N \delta(N)=0$ for all operators $N \in \operatorname{Alg} \mathcal{N}$ with $N^{2}=0$. If $X_{-} \neq X$, then, 
(1) for any $x \in X$ and $f \in X_{-}^{\perp}$, we have $\delta(x \otimes f) \operatorname{ker}(f) \subseteq \operatorname{span}\{x\}$;

(2) there exist linear transformations $B: X \rightarrow X$ and $C: X_{-}^{\perp} \rightarrow X^{*}$ such that $\delta(x \otimes f)=B x \otimes f+x \otimes C f$ and $\langle B x, f\rangle+\langle x, C f\rangle=0$ for all $x \in X$ and $f \in X_{-}^{\perp}$.

Lemma 2.6. Let $\mathcal{N}$ be a nest on a real or complex Banach space $X$ with $\operatorname{dim} X \geq 2$. Assume that $\delta: A \lg \mathcal{N} \rightarrow \operatorname{Alg} \mathcal{N}$ is a linear map satisfying $\delta(P)=\delta(P) P+P \delta(P)$ for all idempotent operators $P \in \operatorname{Alg} \mathcal{N}$ and $\delta(N) N+$ $N \delta(N)=0$ for all operators $N \in \operatorname{Alg} \mathcal{N}$ with $N^{2}=0$. If $\{0\} \neq\{0\}_{+}$, then

(1) for any $f \in X^{*}$ and any $x \in\{0\}_{+}$, we have $\delta(x \otimes f)^{*}\left(\operatorname{ker} x^{* *}\right) \subseteq$ $\operatorname{span}\{f\}$;

(2) there exist linear transformations $B: X^{*} \rightarrow X^{*}$ and $C: \kappa\left(\{0\}_{+}\right) \rightarrow X^{* *}$ such that $\delta(x \otimes f)^{*}=B f \otimes x^{* *}+f \otimes C x^{* *}$ and $\left\langle B f, x^{* *}\right\rangle+\left\langle f, C x^{* *}\right\rangle=0$ holds for all $x \in\{0\}_{+}$and $f \in X^{*}$.

Lemma 2.7. Let $X$ be a Banach space over the real or complex field $\mathbb{F}$. Suppose that $\mathcal{N}$ is a nest on $X$ and $\delta: \operatorname{Alg} \mathcal{N} \rightarrow \operatorname{Alg} \mathcal{N}$ is a linear map satisfying $\delta(P)=\delta(P) P+P \delta(P)$ for all idempotent operators $P \in A \lg \mathcal{N}$ and $\delta(N) N+N \delta(N)=0$ for all operators $N \in \operatorname{Alg} \mathcal{N}$ with $N^{2}=0$. If $X_{-}=X$ and $\{0\}=\{0\}_{+}$, then

(1) there exists a bilinear functional $\beta:\left(\mathcal{D}_{1}(\mathcal{N}) \times \mathcal{D}_{2}(\mathcal{N})\right) \cap \operatorname{Alg} \mathcal{N} \rightarrow \mathbb{F}$ such that $\left(\delta(x \otimes f)-\beta_{x, f} I\right) \operatorname{ker}(f) \subseteq \operatorname{span}\{x\}$ holds for all $x \otimes f \in \operatorname{Alg} \mathcal{N}$;

(2) there exist linear transformations $B: \mathcal{D}_{1}(\mathcal{N}) \rightarrow \mathcal{D}_{1}(\mathcal{N})$ and $C$ : $\mathcal{D}_{2}(\mathcal{N}) \rightarrow \mathcal{D}_{2}(\mathcal{N})$ such that $\delta(x \otimes f)-\beta_{x, f} I=x \otimes C f+B x \otimes f$ holds for all $x \otimes f \in \operatorname{Alg} \mathcal{N}$.

\section{Proof of the main result}

In this section we complete the proof of Theorem 1.1 by using the lemmas in the previous section.

Proof of Theorem 1.1. The "if "part is obvious. We give the proof of the "only if" part here.

Assume that $\mathcal{S} \subset \operatorname{Alg} \mathcal{N}$ is a subset with $\operatorname{span}\{\operatorname{ran}(Z): Z \in \mathcal{S}\}$ is dense in $X$, and $\delta: \operatorname{Alg} \mathcal{N} \rightarrow \operatorname{Alg} \mathcal{N}$ is a linear map derivable on $\mathcal{S}$. Let us show that $\delta$ is a derivation.

By Lemma 2.1, for any $Z \in \mathcal{S}$, we have $\delta(I) Z=0$. As the linear manifold spanned by the ranges of all $Z \in \mathcal{S}$ is dense in $X$, we must have

$$
\delta(I)=0 .
$$

It follows from Lemma $2.2(1)$ and Lemma 2.4 (1) that the following statements are true:

For any $N \in \operatorname{Alg} \mathcal{N}$ with $N^{2}=0$, we have

$$
\delta(N) N+N \delta(N)=0
$$


and

$$
\delta(N Z)=\delta(N) Z+N \delta(Z) \quad \forall Z \in \mathcal{S}
$$

for any idempotent operators $P \in \operatorname{Alg} \mathcal{N}$, we have

$$
\delta(P)=\delta(P) P+P \delta(P)
$$

and

$$
\delta(P Z)=\delta(P) Z+P \delta(Z) \quad \forall Z \in \mathcal{S} .
$$

We consider three cases.

Case 1. $X_{-} \neq X$.

In this case, by Eqs.(3.2) and (3.4), $\delta$ satisfies the hypotheses of Lemmas 2.5. So, there exist linear transformations $B: X \rightarrow X$ and $C: X_{-}^{\perp} \rightarrow X^{*}$ such that $\delta(x \otimes f)=B x \otimes f+x \otimes C f$ and $\langle B x, f\rangle+\langle x, C f\rangle=0$ for all $x \in X$ and $f \in X_{-}^{\perp}$. Thus, as $x \otimes f$ is either a multiple of a idempotent or a square zero operator, by Eqs.(3.3) and (3.5), and the linearity of $\delta$, for any $Z \in \mathcal{S}$, we have

$$
\delta(x \otimes f Z)=(B x \otimes f) Z+(x \otimes C f) Z+(x \otimes f) \delta(Z) .
$$

Note that, for any $T$ and any $x \otimes f \in A \lg \mathcal{N}$, there exists some $\lambda \in \mathbb{C}$ such that $|\lambda|>\|T\|$ and $\left\|(\lambda I-T)^{-1} x\right\|\|f\|<1$. Then both $\lambda I-T$ and $\lambda I-T-$ $x \otimes f=(\lambda I-T)\left(I-(\lambda I-T)^{-1} x \otimes f\right)$ are invertible with their inverses still in $\operatorname{Alg} \mathcal{N}$. It is obvious that $\left(I-(\lambda I-T)^{-1} x \otimes f\right)^{-1}=I+(1-\alpha)^{-1}(\lambda I-T)^{-1} x \otimes f$, where $\alpha=\left\langle(\lambda I-T)^{-1} x, f\right\rangle$.

Thus, for any $T$ and any $x \otimes f \in \operatorname{Alg} \mathcal{N}$ with $x \in X$ and $f \in X_{-}^{\perp}$, take $\lambda \in \mathbb{C}$ such that $|\lambda|>\|T\|$ and $\left\|(\lambda I-T)^{-1} x\right\|\|f\|<1$. By Lemma 2.3, Eqs.(3.1) and (3.6), we get

$$
\begin{aligned}
\delta(Z)= & \delta(\lambda I-T-x \otimes f)\left(I+(1-\alpha)^{-1}(\lambda I-T)^{-1} x \otimes f\right)(\lambda I-T)^{-1} Z \\
& +(\lambda I-T-x \otimes f) \delta\left(\left(I+(1-\alpha)^{-1}(\lambda I-T)^{-1} x \otimes f\right)(\lambda I-T)^{-1} Z\right) \\
= & \delta(Z)-(1-\alpha)^{-1} B\left(x \otimes\left(\lambda I-T^{*}\right)^{-1} f\right) Z \\
& -(1-\alpha)^{-1} \delta(T)(\lambda I-T)^{-1}\left(x \otimes\left(\lambda I-T^{*}\right)^{-1} f\right) Z \\
& -\left(x \otimes\left(\lambda I-T^{*}\right)^{-1} C f\right) Z+\left(x \otimes C\left(\lambda I-T^{*}\right)^{-1} f\right) Z \\
& +(1-\alpha)^{-1}(\lambda I-T) B(\lambda I-T)^{-1}\left(x \otimes\left(\lambda I-T^{*}\right)^{-1} f\right) Z \\
& -\left(x \otimes\left(\lambda I-T^{*}\right)^{-1} \delta(T)^{*}\left(\lambda I-T^{*}\right)^{-1} f\right) Z \\
& -(1-\alpha)^{-1}\left(\left\langle(\lambda I-T)^{-1} x, C f\right\rangle\right. \\
& \left.+\left\langle B(\lambda I-T)^{-1} x, f\right\rangle\right)\left(x \otimes\left(\lambda I-T^{*}\right)^{-1} f\right) Z .
\end{aligned}
$$

As $\left\langle(\lambda I-T)^{-1} x, C f\right\rangle+\left\langle B(\lambda I-T)^{-1} x, f\right\rangle=0$, the above equation becomes

$$
\begin{aligned}
0= & (1-\alpha)^{-1} B\left(x \otimes\left(\lambda I-T^{*}\right)^{-1} f\right) Z \\
& +(1-\alpha)^{-1} \delta(T)(\lambda I-T)^{-1}\left(x \otimes\left(\lambda I-T^{*}\right)^{-1} f\right) Z \\
& +\left(x \otimes\left(\lambda I-T^{*}\right)^{-1} C f\right) Z-\left(x \otimes C\left(\lambda I-T^{*}\right)^{-1} f\right) Z \\
& -(1-\alpha)^{-1}(\lambda I-T) B(\lambda I-T)^{-1}\left(x \otimes\left(\lambda I-T^{*}\right)^{-1} f\right) Z \\
& +\left(x \otimes\left(\lambda I-T^{*}\right)^{-1} \delta(T)^{*}\left(\lambda I-T^{*}\right)^{-1} f\right) Z .
\end{aligned}
$$


Since the $\operatorname{span}\{\operatorname{ran}(Z): Z \in \mathcal{S}\}$ is dense in $X$, we must have

$$
\begin{aligned}
0= & (1-\alpha)^{-1} B x \otimes\left(\lambda I-T^{*}\right)^{-1} f \\
& +(1-\alpha)^{-1} \delta(T)(\lambda I-T)^{-1} x \otimes\left(\lambda I-T^{*}\right)^{-1} f \\
& +x \otimes\left(\lambda I-T^{*}\right)^{-1} C f-x \otimes C\left(\lambda I-T^{*}\right)^{-1} f \\
& -(1-\alpha)^{-1}(\lambda I-T) B(\lambda I-T)^{-1} x \otimes\left(\lambda I-T^{*}\right)^{-1} f \\
& +x \otimes\left(\lambda I-T^{*}\right)^{-1} \delta(T)^{*}\left(\lambda I-T^{*}\right)^{-1} f,
\end{aligned}
$$

and so

$$
\begin{aligned}
& {\left[\delta(T)(\lambda I-T)^{-1}-(\lambda I-T) B(\lambda I-T)^{-1}+B\right] x \otimes\left(\lambda I-T^{*}\right)^{-1} f } \\
= & x \otimes(1-\alpha)\left[C\left(\lambda I-T^{*}\right)^{-1}-\left(\lambda I-T^{*}\right)^{-1} C\right. \\
& \left.-\left(\lambda I-T^{*}\right)^{-1} \delta(T)^{*}\left(\lambda I-T^{*}\right)^{-1}\right] f
\end{aligned}
$$

holds for every $x \in X$. This entails that there is a scalar $\beta_{\lambda}$ such that

$$
\delta(T)(\lambda I-T)^{-1}-(\lambda I-T) B(\lambda I-T)^{-1}+B=\beta_{\lambda} I
$$

on $X$. It follows that

$$
\delta(T)=B T-T B+\beta_{\lambda}(\lambda I-T)
$$

Taking different $\lambda_{1}, \lambda_{2}$ in the above equation with $T \neq I$, we see that, $\left(\lambda_{1} \beta_{\lambda_{1}}-\right.$ $\left.\lambda_{2} \beta_{\lambda_{2}}\right) I=\left(\beta_{\lambda_{1}}-\beta_{\lambda_{2}}\right) T$, which implies that $\beta_{\lambda_{1}}=\beta_{\lambda_{2}}=0$. Consequently, $\delta(T)=B T-T B$ holds for all $T \in \operatorname{Alg} \mathcal{N}$, that is, $\delta$ is a derivation.

Case 2. $\{0\} \neq\{0\}_{+}$.

In this case, $\delta$ satisfies the assumptions in Lemmas 2.6. A similar argument of Case 1 shows that $\delta$ is a derivation, too, and we omit the details.

Case 3. $\{0\}=\{0\}_{+}$and $X_{-}=X$.

In this case $X$ is infinite dimensional and every nonzero element $N$ in $\mathcal{N}$ is infinite dimensional.

By Lemmas 2.7, there exists a bilinear functional $\beta:\left(\mathcal{D}_{1}(\mathcal{N}) \times \mathcal{D}_{2}(\mathcal{N})\right) \cap$ $\operatorname{Alg} \mathcal{N} \rightarrow \mathbb{C}$, linear transformations $B: \mathcal{D}_{1}(\mathcal{N}) \rightarrow \mathcal{D}_{1}(\mathcal{N})$ and $C: \mathcal{D}_{2}(\mathcal{N}) \rightarrow$ $\mathcal{D}_{2}(\mathcal{N})$ such that

$$
\left(\delta(x \otimes f)-\beta_{x, f} I\right) \operatorname{ker}(f) \subseteq \operatorname{span}\{x\}
$$

and

$$
\delta(x \otimes f)-\beta_{x, f} I=x \otimes C f+B x \otimes f
$$

hold for all $x \otimes f \in \operatorname{Alg} \mathcal{N}$. Then, by Eqs.(3.3)-(3.5), Lemma 2.3 and the linearity of $\delta$, for any $Z \in \mathcal{S}$, we have

$$
\delta(x \otimes f Z)=(B x \otimes f) Z+(x \otimes C f)+\beta_{x, f} Z+(x \otimes f) \delta(Z) .
$$



that

Let $\tilde{\beta}_{x, f}=\langle x, C f\rangle+\langle B x, f\rangle$. Then a similar argument as that in [12] shows

$$
\begin{cases}\tilde{\beta}_{x, f}=0, & \text { if }\langle x, f\rangle \neq 0 \\ \tilde{\beta}_{x, f}=-2 \beta_{x, f}, & \text { if }\langle x, f\rangle=0 .\end{cases}
$$

For any $T$ and $x \otimes f \in \operatorname{Alg} \mathcal{N}$, take $\lambda$ such that $|\lambda|>\|T\|$ and $\|(\lambda I-$ $T)^{-1} x\|\| f \|<1$. Note that

$$
I=(\lambda I-T-x \otimes f)\left(I+(1-\alpha)^{-1}(\lambda I-T)^{-1} x \otimes f\right)(\lambda I-T)^{-1},
$$

where $\alpha=\left\langle(\lambda I-T)^{-1} x, f\right\rangle$.

Since $\delta$ is derivable at $Z \in \mathcal{S}$, by Lemma 2.3, Eqs.(3.3) and (3.5), we have

$$
\begin{aligned}
\delta(Z)= & \delta(\lambda I-T-x \otimes f)\left[I+(1-\alpha)^{-1}(\lambda I-T)^{-1} x \otimes f\right](\lambda I-T)^{-1} Z \\
& +(\lambda I-T-x \otimes f) \delta\left(\left(I+(1-\alpha)^{-1}(\lambda I-T)^{-1} x \otimes f\right)(\lambda I-T)^{-1} Z\right) \\
= & {\left[-\delta(T)-B x \otimes f-x \otimes C f-\beta_{x, f} I\right]\left[(\lambda I-T)^{-1} Z\right.} \\
& \left.+(1-\alpha)^{-1}(\lambda I-T)^{-1}(x \otimes f)(\lambda I-T)^{-1} Z\right] \\
& +[\lambda I-T-x \otimes f]\left[(\lambda I-T)^{-1} \delta(Z)+(\lambda I-T)^{-1} \delta(T)(\lambda I-T)^{-1} Z\right. \\
& +(1-\alpha)^{-1}\left(B(\lambda I-T)^{-1}(x \otimes f)(\lambda I-T)^{-1} Z\right. \\
& \left.\left.+(\lambda I-T)^{-1} x \otimes\left((\lambda I-T)^{-1}\right)^{*} f \delta(Z)+\beta_{(\lambda I-T)^{-1} x,\left((\lambda I-T)^{-1}\right)^{*} f} Z\right)\right] .
\end{aligned}
$$

Note that $(1-\alpha)^{-1} \alpha=(1-\alpha)^{-1}-1$ and $\tilde{\beta}_{x, f}=\langle x, C f\rangle+\langle B x, f\rangle$. The above equation gives

$$
\begin{aligned}
& \beta_{x, f}(\lambda I-T)^{-1} Z-(1-\alpha)^{-1} \beta_{(\lambda I-T)^{-1} x,\left(\lambda I-T^{*}\right)^{-1} f}(\lambda I-T) Z \\
= & (1-\alpha)^{-1} \delta(\lambda I-T)\left((\lambda I-T)^{-1} x \otimes\left(\lambda I-T^{*}\right)^{-1} f\right) Z \\
& -(1-\alpha)^{-1}\left(B x \otimes\left(\lambda I-T^{*}\right)^{-1} f\right) Z-\left(x \otimes\left(\lambda I-T^{*}\right)^{-1} C f\right) Z \\
& -(1-\alpha)^{-1} \tilde{\beta}_{(\lambda I-T)^{-1} x, f}\left(x \otimes\left(\lambda I-T^{*}\right)^{-1} f\right) Z \\
& -(1-\alpha)^{-1} \beta_{x, f}\left((\lambda I-T)^{-1} x \otimes\left(\lambda I-T^{*}\right)^{-1} f\right) Z \\
& +\left(x \otimes f(\lambda I-T)^{-1} \delta(\lambda I-T)(\lambda I-T)^{-1}\right) Z+\left(x \otimes C\left(\lambda I-T^{*}\right)^{-1} f\right) Z \\
& +(1-\alpha)^{-1}\left((\lambda I-T) B(\lambda I-T)^{-1} x \otimes\left(\lambda I-T^{*}\right)^{-1} f\right) Z \\
& -(1-\alpha)^{-1} \beta_{(\lambda I-T)^{-1} x,\left(\lambda I-T^{*}\right)^{-1} f}(x \otimes f) Z .
\end{aligned}
$$

Since the linear span of $\{\operatorname{ran}(Z): Z \in \mathcal{S}\}$ is dense in $X$, the above equation implies that

$$
\begin{aligned}
& \beta_{x, f}(\lambda I-T)^{-1}-(1-\alpha)^{-1} \beta_{(\lambda I-T)^{-1} x,\left(\lambda I-T^{*}\right)^{-1} f}(\lambda I-T) \\
= & (1-\alpha)^{-1} \delta(\lambda I-T)(\lambda I-T)^{-1} x \otimes\left(\lambda I-T^{*}\right)^{-1} f \\
& -(1-\alpha)^{-1} B x \otimes\left(\lambda I-T^{*}\right)^{-1} f-x \otimes\left(\lambda I-T^{*}\right)^{-1} C f \\
& -(1-\alpha)^{-1} \tilde{\beta}_{(\lambda I-T)^{-1} x, f} \otimes\left(\lambda I-T^{*}\right)^{-1} f \\
& -(1-\alpha)^{-1} \beta_{x, f}(\lambda I-T)^{-1} x \otimes\left(\lambda I-T^{*}\right)^{-1} f \\
& +x \otimes f(\lambda I-T)^{-1} \delta(\lambda I-T)(\lambda I-T)^{-1}+x \otimes C\left(\lambda I-T^{*}\right)^{-1} f \\
& +(1-\alpha)^{-1}(\lambda I-T) B(\lambda I-T)^{-1} x \otimes\left(\lambda I-T^{*}\right)^{-1} f \\
& -(1-\alpha)^{-1} \beta_{(\lambda I-T)^{-1} x,\left(\lambda I-T^{*}\right)^{-1} f}(x \otimes f) .
\end{aligned}
$$


By a corresponding argument as in the proof of the main result in [12], we see that $\beta_{x, f}=0$ for any $x \otimes f \in \operatorname{Alg} \mathcal{N}$. Then by Eq.(3.7), we get that

$$
\delta(x \otimes f)=x \otimes C f+B x \otimes f \quad \text { holds for all } \quad x \otimes f \in \operatorname{Alg} \mathcal{N} .
$$

Now, a similar argument as in [12] ensures that $\delta$ is a derivation.

Therefore, every subset $\mathcal{S} \subset \operatorname{Alg} \mathcal{N}$ satisfying $\operatorname{span}\{\operatorname{ran}(Z): Z \in \mathcal{S}\}$ is dense in $X$ is an all-derivable subset of $\operatorname{Alg} \mathcal{N}$.

The fact that every subset $\mathcal{S} \subset \operatorname{Alg} \mathcal{N}$ satisfying $\cap\{\operatorname{ker} Z: Z \in \mathcal{S}\}=\{0\}$ is also an all-derivable subset of $A \lg \mathcal{N}$ can be proved similarly by multiplying $Z \in \mathcal{S}$ from the left sides and then applying Lemmas.

The proof of Theorem 1.1 is completed.

\section{References}

[1] R-L. An, J-C. Hou, Characterizations of derivations on triangular ring: Additive maps derivable at idempotents, Lin. Alg. Appl., 431 (2009), 10701080 .

[2] R. L. Crist, Local derivations on operator algebras, J. Func. Anal., 135 (1996), 76-92.

[3] K. R. Davision, Nest Algebras, Pitman Research Notes in Mathematics Series, vol. 191, Longman Scientific and Technical, Burnt mill Harlow, Essex, UK. 1988.

[4] J-C. Hou, X-F. Qi, Additive maps derivable at some point on $\mathcal{J}$-subspace lattice algebras, Lin. Alg. Appl., 429 (2008), 1851-1863.

[5] R. V. Kadison, Local derivations, J. Algebra, 130 (1990), 494-509.

[6] D. R. Larson, A. R. Sourour, Local derivations and local automorphisms of $\mathcal{B}(X)$, Proc. Sympo. in Pure Math., 51 (1990), 187-194.

[7] J. Li, Z. Pan, On derivable mappings, J. Math. Anal. Appl., 374 (2011), 311-322.

[8] X-F. Qi, J-C. Hou, Characterizations of derivations of Banach space nest algebras: All-derivable points, Lin. Alg.. Appl., 432 (2010), 3183-3200.

[9] X-F. Qi, J-C. Hou, Full-derivable points of $\mathcal{J}$-subspace lattice algebras, Rocky Mountain J. Math., to appear.

[10] P. Šemrl, Local automorphisms and derivations on $\mathcal{B}(H)$, Proc. Amer. Math. Soc., 125 (1997), 2667-2680. 
[11] M. Spivac, Derivations and nest algebras on Banach spaces, Israel. J. Math., 50(2) (1985), 193 - 200.

[12] Y-F. Zhang, J-C. Hou, X-F. Qi, Characterizing derivations for any nest algebras on Banach spaces by their behaviors at an injective operator, arXiv:1311.5276 [math. FA] 2013.

[13] J. Zhou, Linear mappings derivable at some nontrivial elements, Lin. Alg. Appl., 435 (2011), 1972-1986.

[14] J. Zhu, C. Xiong, R. Zhang, All-derivable points in the algebra of all upper triangular matrices, Lin. Alg. Appl., 429(4) (2008), 804-818.

[15] J. Zhu, C. Xiong, Derivable mappings at unit operator on nest algebras, Lin. Alg. Appl., 422 (2007), 721-735.

[16] J. Zhu, S. Zhao, Characterization of all-derivable points in nest algebras, Proc. Amer. Math. Soc., 141 (2013), 2343-2350.

Received: November 25, 2013 